\title{
Scale-free networks with an exponent less than two
}

\author{
Hamed Seyed-allaei, ${ }^{1}$ Ginestra Bianconi, ${ }^{2,3}$ and Matteo Marsili ${ }^{2}$ \\ ${ }^{1}$ International School for Advanced Studies, via Beirut 4, 34014 Trieste, Italy \\ ${ }^{2}$ The Abdus Salam International Center for Theoretical Physics, Strada Costiera 11, 34014 Trieste, Italy \\ ${ }^{3}$ INFM, UdR Trieste, via Beirut 2-4, 34014, Trieste, Italy
}

\begin{abstract}
We study scale free simple graphs with an exponent of the degree distribution $\gamma$ less than two. Generically one expects such extremely skewed networks - which occur very frequently in systems of virtually or logically connected units - to have different properties than those of scale free networks with $\gamma>2$ : The number of links grows faster than the number of nodes and they naturally posses the small world property, because the diameter increases by the logarithm of the size of the network and the clustering coefficient is finite. We discuss a simple prototype model of such networks, inspired by real world phenomena, which exhibits these properties and allows for a detailed analytical investigation.
\end{abstract}

PACS numbers: 89.75.-k, 89.75.Da, 89.75.Hc, 89.75.Fb

There has been a recent surge of interest on the network structure which underlie many real world phenomena [1]. This is partly because network's topology plays a key role in their understanding and partly because of the ubiquity of few generic features such as the small world property [2] and scale-free distribution of degrees [3]. The latter has been observed for example in the World Wide Web [4], Citation network [5], Protein Interaction Network [6], film actors [2], electronic circuits [7]. Indeed in each of these systems nodes - web pages or actors - are linked - by hyperlinks or collaboration in the same movie - to a number $k$ of other nodes, which is called the degree of the node [27], and which obeys a power law distribution $P(k) \sim k^{-\gamma}$. In many cases (table【) the exponent $\gamma$ of such a distribution is larger than two which its occurrence has been related to some interaction mechanism such as preferential attachment [3] - in simplified models.

Scale-free networks with an exponent $\gamma<2$ have received less attention, despite of their widespread appearance (table \), in the peer-to-peer Gnutella network 28. 8, 9], outgoing E-mails network [10], traffic in networks 11], co-authorship network in high energy physics 12 and in the network of dependency among software packages [13, 14].

The aim of this letter is to show that simple graphs with $\gamma<2$ have markedly different properties than simple graphs with $\gamma>2$. We shall do this first on the basis of general arguments and then using a prototype model motivated by the above mentioned real networks. This model reproduces all the discussed generic properties. Furthermore we show that its generalization to a weighted network exhibits non-trivial statistical properties.

Generic properties - We focus on simple graphs with uncorrelated degree distribution. In the ensemble of Ref. 15], where the probability of a link between nodes $i$ and $j$ is $p_{i, j}=1-e^{k_{i} k_{j} /(n\langle k\rangle)}$, where $\langle k\rangle=\sum_{i} k_{i} / n$ is the average degree, nodes with degrees $k_{i} \approx \sqrt{n\langle k\rangle}$ cannot be considered as independent. The degrees of a simple scale free graph are uncorrelated only if a structural cutoff $k_{c}(n) \sim \sqrt{n\langle k\rangle}$ is imposed in the degree distribution.
Random uncorrelated networks with $\gamma<2$ differ fundamentally in their topology from networks with $\gamma>2$. Indeed, $\gamma<2$ implies that the average degree increases with the system size $\langle k\rangle \sim n^{\xi}$, which means that the total number of links grows faster than the number of nodes. This in turn means that the cutoff $k_{c}(n)$ diverges with the system size in a non-trivial manner. When $\gamma>2$ the mean degree $\langle k\rangle$ is finite and hence $k_{c}(n) \sim n^{1 / 2}$. On the contrary, for $\gamma<2$ the divergence $\langle k\rangle \sim n^{\xi}$ implies that the structural cutoff scales with system size $n$ as $k_{c}(n) \sim n^{(1+\xi) / 2}$. This and the explicit calculation of $\langle k\rangle$, leads to

$$
\xi=(2-\gamma) / \gamma
$$

Correlated networks with a cutoff $k_{c}(n) \sim n^{\chi}$ which diverges faster with $n$ will exhibit an even faster divergence of $\langle k\rangle$, with $\xi=\chi(2-\gamma)$.

Uncorrelated networks with such a broad distribution of degrees are expected to have a high clustering coefficient. The clustering coefficient is the ratio of number of loops of size 3 [16, 17] to the number of triples of connected nodes, which is $\sum_{i} k_{i}\left(k_{i}-1\right)$. So using Eq. (1) and the fact that $\left\langle k^{2}\right\rangle \sim k_{c}^{3-\gamma}$, we find a finite clustering coefficient:

$$
C \sim \frac{\langle k(k-1)\rangle^{2}}{\langle k\rangle^{3} n} \sim \text { const. }
$$

By contrast, the same argument implies a vanishing clustering coefficient $C \sim n^{2-\gamma}$ for $\gamma>2$.

Such a high clustering is consistent with the presence of a high density core: Indeed a finite fraction of nodes are within a distance $\log \log n$ one from the other [18]. Still the diameter of the network is of order $\log n$. Indeed there is a finite number of nodes with degree $k_{i}=1$ and 2 and these form chains which connect to the core, whose length is exponentially distributed. Hence the longest chain has length $\ell_{\max } \sim \log n$, and it dominates the behavior of the diameter. Similar arguments were also used in reference [18] for graphs with $\gamma>2$. 
The model - Here we study in detail a prototype model of networks with $\gamma<2$ motivated by the real systems discussed above. Our model is based on the idea of aggregation [19] and it is very similar to one recently and independently introduced in Ref. [20, 21] in a different context, and analyzed partly by Alava and Dorogovtsev [22]. We show that its statistical properties can be fully understood analytically and that they reproduce successfully the properties observed in real world networks with $\gamma<2$. Furthermore the model shows that, in networks with $\gamma<2$, the statistics of strength of weighted networks can be highly non-trivial and very different from its counterpart in networks with $\gamma>2$. Therefore, we hope the model may serve as a starting point to understand more complex cases as well as to address different issues, such as the efficiency of search algorithms [23], routing, traffic flow 11] and transmission of infections on peer-to-peer networks.

We consider a network of $n$ nodes and, in each time step, we perform the following two steps:

1. Creation: We create a new node and connect it to a randomly chosen node.

2. Merging: We merge two randomly selected nodes. If the two nodes were already connected, the corresponding link is removed. Likewise we remove multiple links with common neighbors of the two nodes.

The first move is like creating a new software package, e-mail address or running a new instance of Gnutella. The second move can be related to merging two packages or abandoning one in the favor of another, merging two e-mail accounts or shutting down a Gnutella client-server and giving its load to another one.

The model describes a stationary network with a fixed number of nodes. If the second process is run at a smaller rate than the first, the model describes a growing network (see Ref. 22] where a similar extension has been analyzed). Actually, to perform our simulation, we started from a graph with a couple of nodes, then we permitted it to grow by allowing more creation than merging until it reached a given size. After that we merged and created nodes sequentially to keep the number of nodes fixed and we continued it until the system reached the stationary state of the average degree. At that point we started taking snapshots of the network with a given interval that was enough to give us a thousands of independent structures. The interval between sampling was about the same time as we had waited to reach the stationary state. We repeated the process for different network sizes. Results are reported in figure 1 and table \ compares the characteristics of our networks to the one of real world cases. The degree distribution $P(k)$ follows a scaling function of the form $P(k)=k^{-\gamma} f\left(k / n^{\sigma}\right)$ with $\gamma \simeq 1.5$ and $\sigma \simeq 0.67$ where $n$ indicates the total number of nodes and $k$ the degree of the nodes. Here $f(x)$ is a scaling function with $f(x) \sim$ const when $x \ll 1$ and with $f(x)$ decaying faster

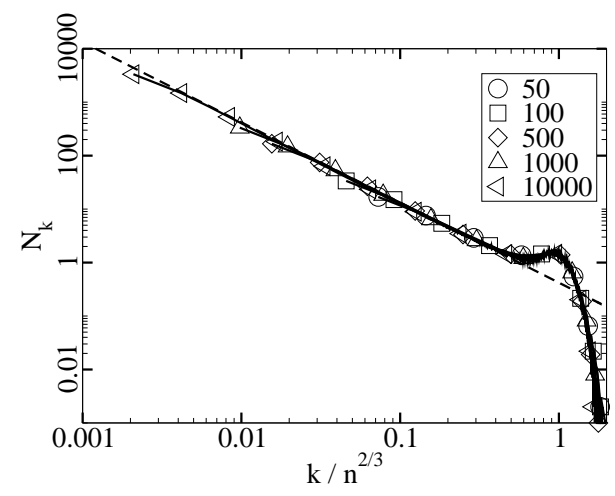

FIG. 1: Collapse plot of degree distribution for networks of different size. The dashed line corresponds to a power law with exponent $-3 / 2$.

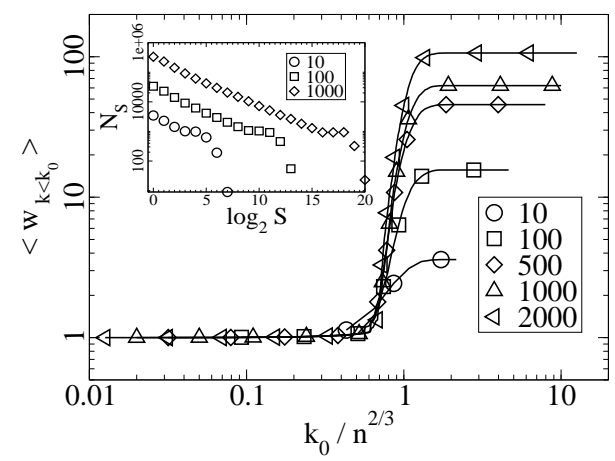

FIG. 2: Main figure shows the average of weight of links that are connected to nodes with a degree less than a given value of $k_{0}$ and the inset shows the histogram of strength of nodes. The legends show the number of nodes in each case.

than any power of $x$ for $x \gg 1$. In our model, since we have an exponent $\gamma<2$ also the total number of links $m$ follows a power-law of the form $m=n^{\xi+1}$ with the exponent $\xi \simeq 0.33>0$, at odd with most studied models with $\gamma>2$ for which $\xi=0$ [3, 16]. The exponents found above agree perfectly with the exponent relations $\sigma=(1+\xi) / 2$ and Eq. (11). Moreover, we found that the networks produced by the above dynamics have the small world properties: their diameter grows as $\log n$ with system size whereas clustering coefficient does not decrease as $n$ increases, in agreement with Eq. (2).

Weighted network - It is also interesting to consider a model of weighted networks with the above dynamics. The idea, for example, is that if the link between two software packages $i$ and $j$ means that package $i$ calls package $j$, it might also be interesting to keep track of how many times $i$ calls $j$. Hence we associate a weight to each link $i j$ and assume that it evolves according to the following rules:

- A fresh link that connects a new node to the network has weight one. 
- When merging two nodes $i$ and $j$ which are both linked to the same node $k$, as before we only keep one link, and its weight is the sum of the weights of the previous links.

In the previous example, when two software packages are merged, the new package inherits all the calls to a third piece of software of the merging packages. Likewise, when two e-mail account are merged, we assume that the traffic of e-mails to a third account will be the sum of the traffic originating from the two accounts before the merge. This neglects the presence of complementarities, which can be an important issue in some cases, but is the most natural way to introduce weights in the model. Weights allow us to define the strength of a node in the usual way 24], i.e. as the sum of the weights of outgoing links.

The sum of all the weights increases when we add a node, and it decreases when we merge two nodes that are connected; therefore, one can expect it to reach the steady state. This was confirmed by simulation, which also shows that the distribution of the strengths decays as a power-law with an exponent 1.5. This would be consistent with a linear relation of strength versus degree, but Fig. 2 shows that such a relation only holds for small $k$ and that most of the weight concentrates on high degree nodes.

Analytic approach - It is possible to shed light on these finding and to calculate the exact value of the exponents for this model, following similar arguments to those of Ref. 22]. We can combine the two operations above in a single one where we replace two nodes $i$ and $j$ by two nodes of which $i$ inherits all the links (incoming and outgoing) of both nodes and $j$ looses all links, and acquires a new link to a randomly chosen new node 29]. If $k_{i}^{\prime}$ and $k_{j}^{\prime}$ are the degrees of the two nodes after the process, we have

$$
\begin{gathered}
k_{i}^{\prime}=k_{i}+k_{j}-m_{i j}-a_{i j} \\
k_{j}^{\prime}=1
\end{gathered}
$$

where $a_{i j}=1$ if the link $i j$ exists and $m_{i j}=\sum_{\ell} a_{i \ell} a_{j \ell}$ is the number of sites who were linked to both $i$ and $j$. Given that $i$ and $j$ are chosen at random, $m_{i j}$ and $a_{i j}$ can be regarded as random variables. The probability that the link $i j$ exists is $\left\langle a_{i j}\right\rangle=k_{i} k_{j} /(n\langle k\rangle)$, likewise the average number of nodes connected to both $i$ and $j$ is

$$
\left\langle m_{i j}\right\rangle=\sum_{\ell} \frac{k_{i} k_{\ell}}{n\langle k\rangle} \frac{k_{\ell} k_{j}}{n\langle k\rangle}=\mu k_{i} k_{j}
$$

where $\mu=\left\langle k^{2}\right\rangle / n\langle k\rangle^{2}$. Let us now introduce the generating function for the degree distribution

$$
\Phi(z)=\frac{1}{N} \sum_{i=1}^{N} E\left(z^{k_{i}}\right) .
$$

In the stationary state, we can use Eq. (3) to derive the equation

$$
\begin{aligned}
\Phi(z) & =\frac{1}{2} E\left(z^{k_{i}+k_{j}-m_{i j}-a_{i j}-a_{j i}}\right)+\frac{1}{2} z \\
& =\frac{1}{2}\left\{z+E\left[z^{k_{i}+k_{j}} e^{\mu k_{i} k_{j} h(z)}\left(1+\eta k_{i} k_{j} h(z)\right)\right]\right\}
\end{aligned}
$$

where $\eta=1 /(n\langle k\rangle), h(z)=(1-z) / z$ and the last equality hinges upon the observation that $m_{i j}$ is a Poisson variable with mean given by Eq. (4) and that $a_{i j}$ is a random bit with $\left\langle a_{i j}\right\rangle=\eta k_{i} k_{j}$. Now we observe that both $\mu$ and $\eta \rightarrow 0$ as $n \rightarrow \infty$, consequently $\Phi(z)$ can be expanded in a power series in $\mu$ and $\eta$. The leading term $(\mu=\eta=0)$ yields $2 \Phi(z)=\Phi^{2}(z)+z$, i.e.

$$
\Phi(z)=1-\sqrt{1-z}=\frac{1}{2 \Gamma(1 / 2)} \sum_{k=1}^{\infty} \frac{\Gamma(k-1 / 2)}{k !} z^{k} ;
$$

Therefore, for $n \rightarrow \infty$, we find $P(k)=\frac{1}{2 \Gamma(1 / 2)} \frac{\Gamma(k-1 / 2)}{k !} \sim$ $k^{-\gamma}$ with $\gamma=3 / 2$. The exponent relations derived earlier can then be used to conclude that $\sigma=2 / 3$ and $\xi=1 / 3$. This conclusion is also supported by a direct calculation of the next terms in the small $\mu$ expansion. These finite $n$ corrections introduce a finite cutoff $k_{c} \sim n^{\sigma}$ in the distribution, but leads to cumbersome formulas which we will not detail here. A further way to compute $\sigma$ comes from observing that the average of Eq. (3) in the stationary state yields $1=\left\langle k^{2}\right\rangle / n+\langle k\rangle / n$, i.e. $\left\langle k^{2}\right\rangle \sim$ $n$. This combined with the relation $\left\langle k^{q}\right\rangle \sim n^{\sigma(q-\gamma+1)}$ implies $\sigma=2 / 3$. This shows that the exponent relations $\sigma=(1+\xi) / 2$ and Eq. (1) - which are valid for random graphs - can be explicitly verified in this model.

A simple argument also allows us to understand the statistics of weights. Indeed at each time step, a new link with weight $w=1$ is added. At the same time, the weight of the link between nodes $i$ and $j$, if present, is removed. In the stationary state, then we expect that the probability $\langle k\rangle / n$ of an existing link to be chosen, times its average weight $\langle w\rangle$ must be equal to one. Hence $\langle w\rangle \sim n /\langle k\rangle \sim n^{2 / 3}$. Unlike for the degree distribution, we do not expect a cutoff in the distribution of weights [30]. Assuming that $P(w) \sim w^{-\eta-1}$ with $\eta<1$, we know that $\langle w\rangle \sim(n\langle k\rangle)^{1 / \eta-1}$. Combining this with $\langle w\rangle \sim n^{2 / 3}$ we find that $\eta=2 / 3$, in perfect agreement with numerical simulations. Concerning the node's strength $s_{i}$, in order to explain the behavior of Fig. 2 it is crucial to observe that nodes with $k_{i} \ll k_{c}$ will have links with weights of order one. Indeed, merge events in which nodes $i$ and $j$ share some of their neighbors are rare if $\left\langle m_{i j}\right\rangle=\left(k_{i} /\langle k\rangle\right)\left(k_{j} /\langle k\rangle\right) \ll 1, k_{i} \ll k_{c}$ or if $k_{j} \ll k_{c}$. We therefore expect that links belonging to nodes with $k_{i} \ll k_{c}$ have weights of order one, i.e. that $s_{i} \sim k_{i}$. For $k_{i} \sim k_{c}$ instead the additive process of weights on links to shared neighbors becomes relevant, eventually leading to a very broad distribution of weights on such nodes. This is a rather non-standard situation compared to that of most weighted networks with $\gamma>2$ [24].

Conclusions - We have discussed the properties of complex scale free networks with degree distribution expo- 
nent $\gamma<2$, which characterizes many real systems. We have shown that these properties are reproduced by a simple prototype model motivated by such real systems. A key characteristic of this class of networks is that their average degree grow with the system size, which suggests that making a link is inexpensive. This is indeed the case for networks of software packages. In fact it is costly to make a package, but it is costless to use an already existing package. Interestingly, a peculiarity of the model is that it involves global moves. This requires some sort of global information exchange mechanisms, that is not part of the network itself, that allows nodes to interact globally. In the example of software packages, this information exchanges happens among programmers, in fact they are responsible for the evolution of the system and they do not exchange information only through the system. While both properties are likely to hold only for open source packages, they might not apply to commercial software, which might be expensive to link to. A further problem is that statistical information on commercial software dependencies is not available. These two features also characterize other networks: for example, in Gnutella each node is a computer. But each link is only a logical connection between two computers and does not require any additional hardware. In the case of Gnutella network there are web caches that store the information of nodes and share them with other nodes but these caches are not considered as a part of the network itself. It is tempting to conjecture that the relation between these two properties and networks with exponent $\gamma<2$ is generic. This, applied to co-authorship network, suggests that global interaction and information diffusion plays an essential role in establishing a dense collaboration network.

We wish to thank S. Dorogovtsev, A. Maritan, J. Banavar for useful discussions. Work partially supported by EVERGROW and by EU grant HPRN-CT-2002-00319, STIPCO.
[1] S. Dorogovtsev and J. F. F. Mendes, Evolution of Networks: From Biological Nets to the Internet and $W W W$ (Oxford University Press, 2003), ISBN 0198515901.

[2] D. J. Watts and S. H. Strogatz, Nature 393, 440 (1998).

[3] A. L. Barabási and R. Albert, Science 286, 509 (1999).

[4] A. Broder, R. Kumar, F. Maghoul, P. Raghavan, S. Rajagopalan, R. Stata, A. Tomkins, and J. Wiener, Computer Networks-the International Journal of Computer and Telecommunications Networking 33, 309 (2000).

[5] S. Redner, European Physical Journal B 4, 131 (1998).

[6] H. Jeong, S. Mason, A. Barabasi, and Z. Oltvai, Nature 411, 41 (2001).

[7] R. Cancho, C. Janssen, and R. Sole, Phys. Rev. E 64, Art. No. 046119 (2001).

[8] URL http://en.wikipedia.org/wiki/Gnutella

[9] M. Jovanovic, F. Annexstein, and K. Berman, Tech. Rep., University of Cincinnati (2001), URL

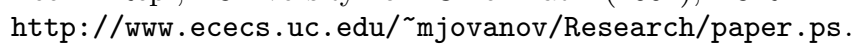

[10] H. Ebel, L. Mielsch, and S. Bornholdt, Phys. Rev. E 66, Art. No. 035103 (2002).

[11] Z. Toroczkai and K. Bassler, Nature 428, 716 (2004).

[12] M. Newman, Phys. Rev. E 64, Art. No. 016131 (2001).

[13] M. Newman, Phys. Rev. E 67, Art. No. 026126 (2003).

[14] S. Valverde and R. V. Sole, ArXiv Condensed Matter e-prints (2003), arXiv:cond-mat/0307278.

[15] M. Molloy and B. Reed, Random Structures and Algorithms 6, 161 (1995).

[16] M. Newman, Siam Review 45, 167 (2003).

[17] G. Bianconi and M. Marsili (2005), cond-mat/0502552.

[18] F. Chung and L. Lu, PNAS 99 (2002).

[19] J. R. Banavar, P. De Los Rios, A. Flammini, N. S. Holter, and A. Maritan, Physical Review E 69, Art. No. 036123 (2004).

[20] B. J. Kim, A. Trusina, P. Minnhagen, and K. Sneppen (2004), nlin.AO/0403006.

[21] P. Minnhagen, M. Rosvall, K. Sneppen, and A. Trusina, Physica A-Statistical Mechanics And Its Applications 340, 725 (2004).

[22] M. J. Alava and S. N. Dorogovtsev, Phys. Rev. E 71,

\begin{tabular}{|l|c|c|c|c|c|}
\hline Network of & $n$ & $m$ & $\gamma$ & $C$ & $l$ \\
\hline Our Simulation & 1000 & 7696 & $3 / 2$ & 0.45 & 3.69 \\
\hline Gnutella [9] & 1026 & 3752 & 1.4 & - & 3.6 \\
\hline $\begin{array}{l}\text { Dependency } \\
\text { of software } \\
\text { packages[16] }\end{array}$ & 1439 & 1723 & $1.6 / 1.4$ & 0.083 & 2.42 \\
\hline E-mails [10] & 59912 & 86300 & 1.8 & - & 4.95 \\
\hline Word web [25] & 478773 & $1.8 \times 10^{7}$ & 1.5 & 0.69 & - \\
\hline $\begin{array}{l}\text { Co-authorship } \\
\text { in HEP [12] }\end{array}$ & 56627 & 9796471 & 1.2 & 0.73 & 4.0 \\
\hline WWW [4] & $2 \times 10^{8}$ & $2 \times 10^{9}$ & $2.1 / 2.7$ & - & 16.8 \\
\hline Internet [26] & 10697 & 31992 & 2.5 & 0.035 & 3.31 \\
\hline PIN [6] & 2115 & 2240 & 2.4 & 0.072 & 6.8 \\
\hline Citation [5] & 783339 & 6716198 & 3.0 & - & - \\
\hline Actors [2] & 449913 & 25516482 & 2.3 & 0.20 & 3.48 \\
\hline $\begin{array}{l}\text { Electronic } \\
\text { Circuits [7] }\end{array}$ & 24097 & 53248 & 3.0 & 0.010 & 11.05 \\
\hline
\end{tabular}

TABLE I: Results of our simulation and its comparison to some empirical observations. In the case of directed network the exponents is shown in the form of in/out. Here the total number of nodes, links, the exponent, clustering coefficient, mean of shortest paths are represented by $n, m, \gamma, C, l$.

036107 (2005).

[23] L. Adamic, R. Lukose, A. Puniyani, and B. Huberman, Phys. Rev. E 64, Art. No. 046135 (2001).

[24] A. Barrat, M. Barthélemy, R. Pastor-Satorras, and A. Vespignani, PNAS 101, 3747 (2004).

[25] R. F. i Cancho and R. V. Solé, Proceedings: Biological Sciences 268, 2261 (2001).

[26] A. Medina, I. Matta, and J. Byers, Computer Communication Review 30, 18 (2000).

[27] By networks we mean simple graphs, those with at most one link between any two nodes and no tadpole. Additional features can be included by the generalization to weighted graphs 24]. 
[28] Gnutella is a file sharing network which operates without a central server: Files are exchanged directly between users, using a proper software.

[29] The discussion which follows refers to an undirected graph, for the sake of simplicity. The same conclusions hold for a directed graph if we disregard the link's direc- tion.

[30] This is because links are randomly drawn. Loosely speaking, there is no "interaction" term in the links' weight process. 